\title{
GEPRO: do Sonho à Realidade
}

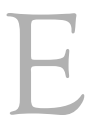

m setembro de 2002, durante o XXIV Congresso Brasileiro de Reumatologia, realizado em Goiânia - GO, a Sociedade Brasileira de Reumatologia lançou um projeto de estudo epidemiológico sobre a esclerose sistêmica no Brasil. O objetivo principal deste estudo é traçar um perfil da doença em nosso país e, de maneira concomitante, contribuir para a criação e para o aperfeiçoamento de centros de estudo da esclerose sistêmica, doença de difícil controle cujo prognóstico, embora ainda necessite melhorar muito, depende do diagnóstico precoce da enfermidade. O referido estudo recebeu o nome de GEPRO (Grupo de Esclerose Sistêmica do Projeto Pronuclear), sendo gerenciado por pesquisadores do Projeto Pronuclear da Sociedade Brasileira de Reumatologia, onde a idéia nasceu e ganhou a sua forma atual, de maneira a poder ser estendida a todos os centros de pesquisa nacionais interessados.

O protocolo GEPRO consta de um questionário de 245 itens com uma única resposta por item, associado ao preenchimento de uma Escala de Gravidade da Doença, segundo critérios de Medsger e colaboradores. Após um período de três meses para avaliação da capacidade de cada centro participante executar os diversos exames que constam no protocolo, o preenchimento oficial iniciou-se no dia $1^{\circ} \mathrm{de}$ janeiro de 2003. O ano de 2003 foi o ano da implementação do protocolo, da viabilização de ambulatórios específicos de esclerodermia, da criação de uma mentalidade comum de avaliar a doença e da aquisição de colaboradores locais para o auxílio no preenchimento do extenso protocolo. Ao final de um ano, 30 centros (relacionados abaixo, com seus coordenadores) estão aptos a participar deste projeto,

\section{GEPRO - LISTA DE CENTROS PARTICIPANTES E SEUS COORDENADORES}

\section{SÃo PAULO}

Universidade Estadual de Campinas (UNICAMP)

Dr. Percival Degrava Sampaio-Barros (Coordenador Nacional)

Dr. João Francisco Marques Neto que não alterará suas linhas de pesquisa específicas em esclerodermia, mas servirá para criar uma rede de pesquisadores interessados em investigar a doença e proporcionar maior qualidade de atendimento aos pacientes necessitados. E a idéia do GEPRO já serviu de estímulo para a criação do GLADES, o Grupo Latino-Americano de Estudo da Esclerodermia, ligado à PANLAR, a Liga Pan-Americana Contra o Reumatismo.

Depois de um ano de trabalho duro, no ano de 2004 começará a fase de divulgação do GEPRO, que se inicia com a publicação deste número especial da Revista Brasileira de Reumatologia, dedicado à esclerose sistêmica, feito com carinho pelos participantes do GEPRO. Estima-se divulgar os primeiros resultados do GEPRO já no XXV Congresso Brasileiro de Reumatologia, a ser realizado no Rio de Janeiro em outubro próximo, que contará com a participação da Prof. ${ }^{a}$ Carol Black, chefe do Serviço de Reumatologia do Royal Free Hospital de Londres, o mais importante centro de pesquisa em esclerodermia na Europa. E outras novidades interessantes estarão programadas para este ano de 2004.

É o sonho se transformando em realidade...

PERCIVAL D. SAMPAIO-BARROS Universidade Estadual de Campinas (UNICAMP) Coordenador Nacional do GEPRO

LUIS EDUARDO C. ANDRADE Universidade Federal de São Paulo (EPM-UNIFESP) Coordenador Nacional do Projeto Pronuclear

Universidade Federal de São Paulo (UNIFESP)

Dr. Luis Eduardo Coelho de Andrade (Coordenador do Projeto Pronuclear)

Dra. Rozana Mesquita Ciconelli (Epidemiologista Clínica)

Universidade de São Paulo (USP)

Dra. Cláudia Lobato Borges

Universidade de São Paulo - Ribeirão Preto (USP-RP)

Dr. Eduardo Antonio Donadi 
Hospital do Servidor Público Estadual

Dr. José Carlos Mansur Szajubok

UNESP - Botucatu

Dr. Oswaldo Melo da Rocha

\section{BAHIA}

Escola Bahiana de Medicina e Saúde Pública

Dr. Mittermayer Santiago

\section{CEARÁ}

Universidade Federal do Ceará

Dra. Sheila Marcia Fontenele

\section{DISTRITO FEDERAL}

Hospital de Base de Brasília

Dr. Gustavo de Paiva Costa

Universidade de Brasília (UnB)

Dr. Cezar Kozak Simaan

\section{GOIÁS}

Universidade Federal de Goiás

Dr. Nilzio Antonio da Silva

MATO GROSSO DO SUL

Universidade Federal de Mato Grosso do Sul

Dr. Izaias Pereira da Costa

\section{MINAS GERAIS}

Hospital Governador Israel Pinheiro - Belo Horizonte

Dra. Christiane Cota Vieira

Universidade Federal de Minas Gerais (UFMG)

Dr. Caio Moreira

Universidade Federal de Uberlândia

Dr. Ben-Hur Braga Taliberti

\section{PARÁ}

Universidade Federal do Pará

Dra. Maria de Fátima Lobato da Cunha Sauma

\section{PARAÍBA}

Universidade Federal da Paraíba

Dra. Eutília Andrade Freire
PARANÁ

Universidade Federal do Paraná (UFPR)

Dr. Sebastião César Radominski

Hospital Evangélico de Curitiba

Dra. Telma Larocca Skare

Universidade Estadual de Londrina (UEL)

Dr. Luis Carlos Lúcio de Carvalho

Dra. Margarida de Fátima Carvalho

Universidade Estadual de Maringá

Dr. Marco Antonio da Rocha Loures

PERNAMBUCO

Universidade Federal de Pernambuco (UFPE)

Dra. Angela Duarte

\section{RIO DE JANEIRO}

Hospital dos Servidores do Estado

Dr. Joaquim Jaguaribe Nava Ribeiro

Universidade do Estado do Rio de Janeiro (UERJ)

Dr. Roger Abramino Levy

Universidade do Rio de Janeiro (UNIRIO)

Dra. Maria Cecília da Fonseca Salgado

Universidade Federal do Rio de Janeiro (UFRJ)

Dr. Mario Newton Azevedo

\section{RIO GRANDE DO NORTE}

Universidade Federal do Rio Grande do Norte (UFRN)

Dra. Maria José Pereira Vilar

RIO GRANDE DO SUL

Universidade Federal do Rio Grande do Sul (UFRGS)

Dr. Ricardo Machado Xavier

PUC - Porto Alegre

Dr. Mauro W. Keiserman

SANTA CATARINA

Universidade Federal de Santa Catarina (UFSC)

Dra. Adriana Fontes Zimmermann 